\title{
Hybrid Element-Free Galerkin-Finite Element Method for Electromagnetic Field Computations
}

\author{
Vlatko Cingoski, Naoki Miyamoto, and Hideo Yamashita
}

\begin{abstract}
A new hybrid computational procedure utilizing the Element-free Galerkin method (EFGM) and the finite element method (FEM) for the electromagnetic field computations is proposed. With the proposed method, only a part of the analyzed domain is modeled using the EFGM, while the rest of the analysis domain is discretized using the ordinary FEM. The connection between both subdomains is enabled using intermediate analysis subdomain where new hybrid shape functions are defined utilizing both EFGM and FEM shape functions as bases. The accuracy of the obtained results by the proposed method is of the same order with those obtained by the ordinary FEM, however, with less computation effects.
\end{abstract}

Index Terms-Element-free Galerkin method, finite element method, magnetostatics, optimization methods.

\section{INTRODUCTION}

$\mathbf{F}$ OR PROBLEMS that have moving objects or objects that constantly change their geometrical appearance during analysis (e.g. inverse shape optimization problems), it is advisable to utilize new solution methods that are based entirely on nodal distribution and does not need generation of any finite element mesh. Recently, we proposed such computation method and it has been shown that it can provide results with the accuracy of the same order as those obtained by means of the FEM [1]. This, so-called Element-free Galerkin method (EFGM) is considered as a meshless method, because to obtain an approximated solution of a problem it requires only the definition of a set of nodes distributed over the entire analysis region and the definition of the boundary conditions. However, as it was pointed out in [1], the EFGM exhibits two major drawbacks: 1 ) it is usually computationally slower than the FEM for the same accuracy of the solution, and 2) it has difficulties for the setting of the boundary conditions, especially those of the Dirichlet type. While the roots for the first problem lie in the needs for a larger number of integration points per each integration cell in order to increase the solution accuracy, the second problem is the direct result of the approximation method used, which for this method is the so-called moving least square method [2]. Recently, few other papers on these topics have appeared, which additionally shows that the interest for the meshless methods is constantly increasing [3], [4].

Manuscript received October 25, 1999.

V. Cingoski is with the Electric Power Company of Macedonia, Skopje, Macedonia (e-mail: vlatko@esmak.com.mk.).

N. Miyamoto and H. Yamashita are with Hiroshima University, Japan (e-mail: yama@eml.hiroshima-u.ac.jp.).

Publisher Item Identifier S 0018-9464(00)06603-6.

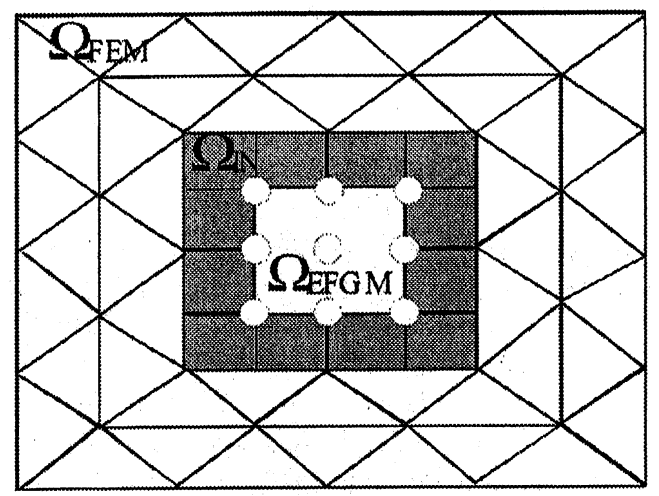

Fig. 1. Typical analysis domain with subdomains.

In order to solve the above described problems of the EFGM, in this paper we propose a new hybrid computation method based on both methods, the EFGM and the FEM. With this new method, only a part of the analysis domain that includes moving objects or objects that change their geometrical shapes during computation is modeled according to the EFGM, while the rest of the analysis domain is discretized into finite elements according to the ordinary FEM. Each analysis subdomain uses its own interpolation procedures and shape functions, and the connection between both subdomains is enabled using the so-called intermediate analysis subdomain where a new hybrid procedure is defined with new hybrid shape functions utilizing both shape functions from the EFGM and the FEM, simultaneously. The main features of the proposed hybrid computation method are:

- By using the common FEM procedure, setting of the boundary conditions (especially the Dirichlet type boundary conditions) is exactly ensured;

- Avoiding remeshing of the changing parts of the analysis domain. Around moving parts or geometrically changing object boundaries an EFGM domain is set and in this area nodal distribution is generated only once;

- By reducing the EFGM analysis domain only to the necessary minimum, the computation time is decreased achieving high accuracy of the results and versatility of the computation procedure.

\section{HYBRID ELEMENT-FREE GALERKIN-FinITE ELEMENT METHOD}

Fig. 1 represents a simplified analyzed domain divided into three analysis subdomains $\Omega_{\mathrm{EFGM}}, \Omega_{\mathrm{FEM}}$, and $\Omega_{\mathrm{IN}}$, which are the EFGM, the FEM and the intermediate subdomain, respectively. It is visible that inside the EFGM subdomain only a set of 


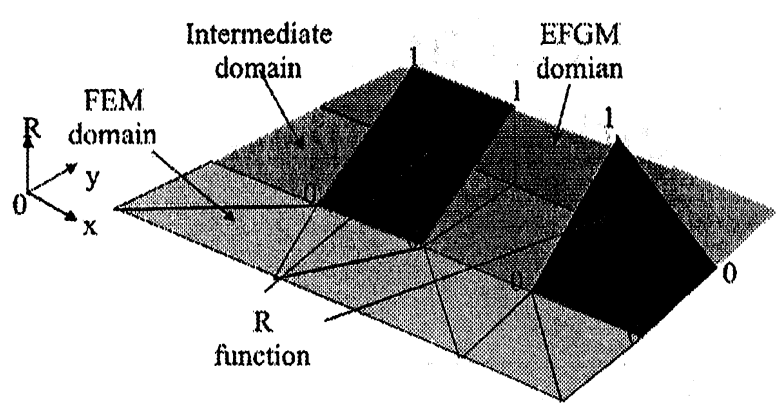

Fig. 2. Shape of the interpolation function $R$.

nodes is defined, while for the FEM subdomain the regular triangular finite element mesh is generated. The intermediate subdomain where also a set of finite elements is defined surrounds the EFGM subdomain. The unknowns are set at each node of the entire domain and the interpolation of the unknown function at arbitrary point $\mathrm{p}$ in accordance with the Galerkin method is $A_{p}=\sum_{i=1}^{k} N_{i} A_{i}$, where $A_{i}$ are the unknown vector potential values at each triangular node for the FEM or a node inside the domain of influence for the EFGM. Each analysis subdomain has different shape functions: $N_{i}^{\mathrm{FEM}}$, for the FEM subdomain, and $N_{i}^{\mathrm{EFGM}}$ for the EFGM subdomain, respectively. Therefore, the above approximation is valid only for each subdomain separately. For point $p$, which lays inside the intermediate subdomain a new hybrid, interpolation procedure is utilized given with this expression

$$
A_{p}=R \sum_{i=1}^{n n} N_{i}^{\mathrm{EFGM}} A_{i}^{\mathrm{EFGM}}+(1-R) \sum_{i=1}^{3} N_{i}^{\mathrm{FEM}} A_{i}^{\mathrm{FEM}}
$$

where $n n$ is the total number of nodes included inside the influence radius of point $p$, while $R$ is a function (see Fig. 2), which changes linearly between the EFGM and the FEM boundary lines $\Gamma^{\mathrm{EFGM}}$ and $\Gamma^{\mathrm{FEM}}$, respectively:

$$
R= \begin{cases}1: & \text { on } \Gamma^{\mathrm{EFGM}} \\ 0: & \text { on } \Gamma^{\mathrm{FEM}}\end{cases}
$$

\section{ApPLiCATIONS}

The proposed Element-free Galerkin method (EFGM) was compared with the FEM for several electromagnetic problems such as:

- magnetostatic test model,

- moving coil model, and

- inverse shape optimization model.

\section{A. Magnetostatic Test Model}

A simple magnetostatic model comprised of a coil placed in the air as shown in Fig. 3, was used for verification the accuracy and the computation speed of the proposed hybrid method. The square shaped $15 \times 15 \mathrm{~mm}$ area including the coil was modeled using EFGM. The open-air domain was modeled using the FEM and these two subdomains were interconnected by the small strip of an intermediate domain as shown schematically

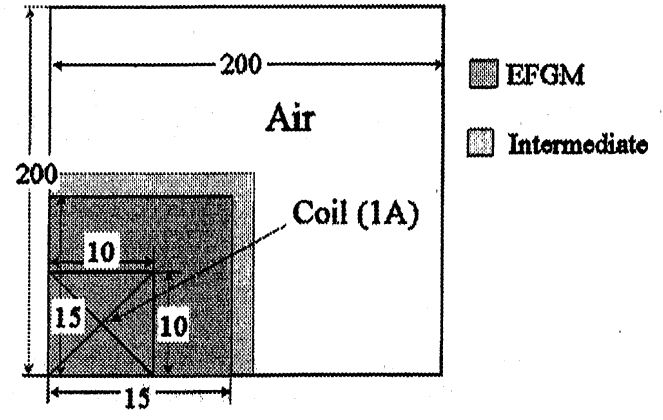

Fig. 3. Application model \#1: Coil in the air.

TABLE I

MODEL \#1: MESH AND COMPUTATION DATA

\begin{tabular}{lrrr}
\hline & $\begin{array}{c}\text { Hybrid } \\
\text { (Case a) }\end{array}$ & $\begin{array}{l}\text { Hybrid } \\
\text { (Case b) }\end{array}$ & FEM \\
\hline $\begin{array}{l}\text { Nodes in } \\
\text { EGFM area }\end{array}$ & 49 & 169 & \\
\hline $\begin{array}{l}\text { Elements in } \\
\text { FEM area }\end{array}$ & 894 & 894 & 992 \\
\hline $\begin{array}{l}\text { Total number } \\
\text { of nodes }\end{array}$ & 540 & 660 & 540 \\
\hline $\begin{array}{l}\text { Computation } \\
\text { Time [sec] }\end{array}$ & 2.1 & 3.2 & 1.6 \\
\hline
\end{tabular}
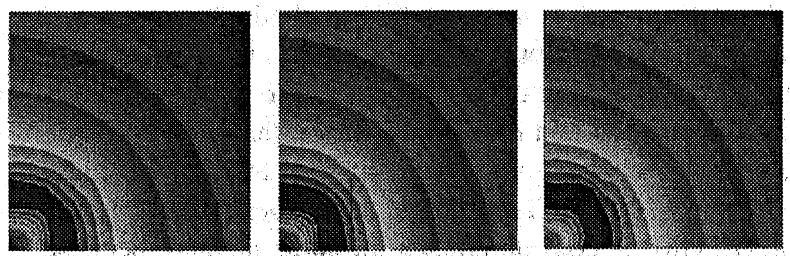

Fig. 4. Comparison between obtained magnetic flux density distributions for the hybrid method and the FEM.

in Fig. 3. For the hybrid method, two separate cases, case $a$, and case $b$ were treated. In Table I the mesh and computation data is given for all three model.

Fig. 4 shows the comparison of the magnetic flux density distributions obtained by the proposed hybrid method and the original FEM. As can be seen, all three distributions are almost identical, which shows that the hybrid method can provide computation results with the same accuracy as the ordinary FEM. This can be further proven using the graph presented in Fig. 5, where we show the relationship between the absolute error of the results and the amount of cumulative area that has that amount of absolute error $\varepsilon$ according to the following equation

$$
C=\frac{\text { number of points with error larger than } \varepsilon}{\text { total number of points }} \times 100[\%]
$$

It is apparent that case $b$ (which has more nodes than the FEM) has better accuracy than the FEM. On the other side, case $a$, with same number of nodes as the FEM has accuracy of the same order as the FEM. Regarding the computation time, the ordinary FEM exhibits fastest solution as shown in Table I. 


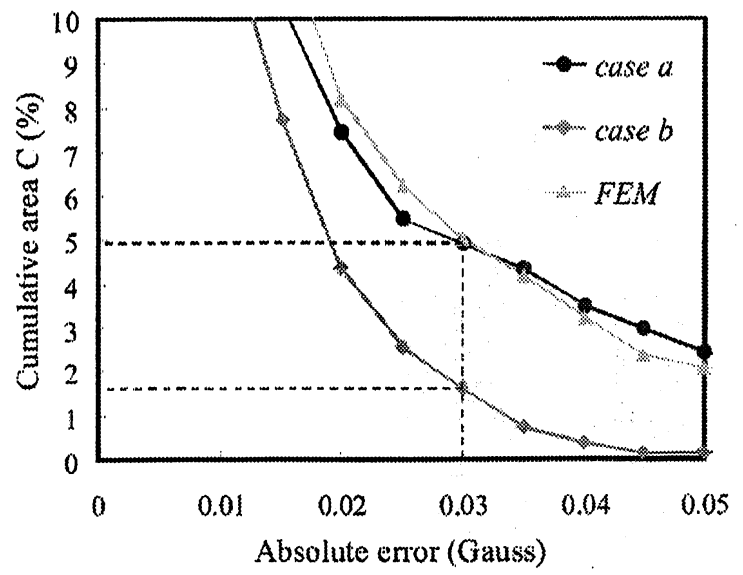

Fig. 5. Comparison of the cumulative area versus absolute error.

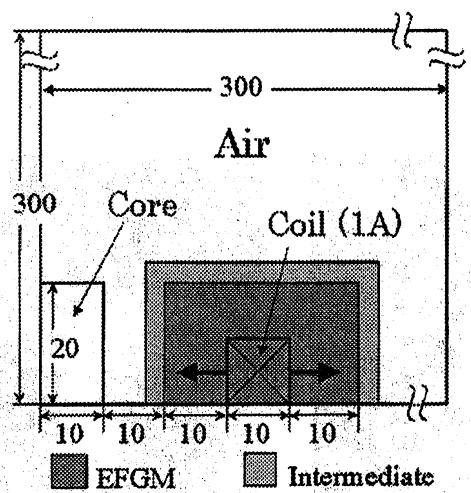

Fig. 6. Application model \#2: Moving coil.

TABLE II

MODEL \#2: MESH AND COMPUTATION DATA

\begin{tabular}{|c|c|c|}
\hline & $\begin{array}{l}\text { Hybrid } \\
\text { Method }\end{array}$ & FEM \\
\hline Nodes in EFGM area & 32 & $\overline{1}$ \\
\hline Elements in FEM area & 1899 & 1995 \\
\hline Total number of nodes & 1057 & 1057 \\
\hline Computation time [sec] & 3.9 & 2.2 \\
\hline
\end{tabular}

\section{B. Moving Coil Model}

As already mentioned in the introduction of this paper, the main purpose for development of the proposed hybrid method was existence of some specific problems where we want to avoid the remeshing of the analysis domain. A model with a moving coil shown in Fig. 6 was considered for testing the effectiveness of the hybrid method. This model consists of a ferrite core and a coil that can move freely left or right along the $x$-axis. In Fig. 6 , the dark gray area represents the EFGM subdomain, the light gray area is the intermediate subdomain. The rest of the analysis domain is the FEM subdomain. The main computation data for both models, for hybrid method and for FEM, respectively, is presented in Table II.

Fig. 7 shows the obtained results for the magnetic flux density distribution with magnetic flux lines for both computation
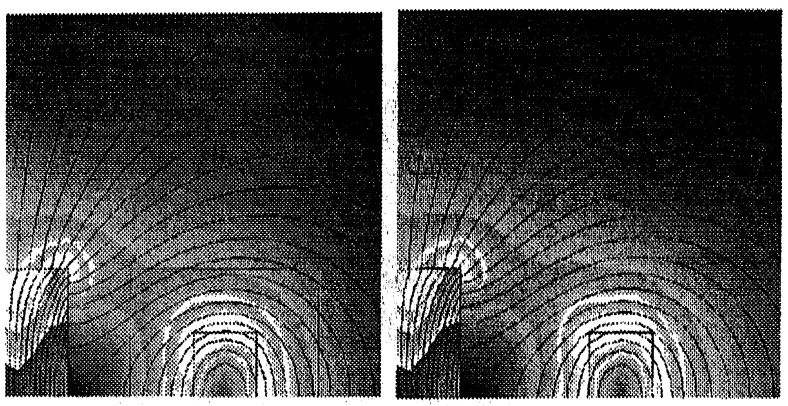

Fig. 7. Results comparison: Model \#2.
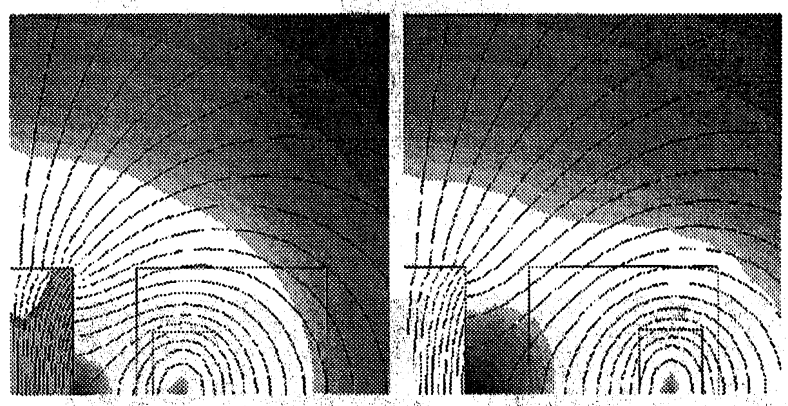

Fig. 8. Computed results using proposed hybrid method for two different coil positions.

methods, the hybrid one and the FEM. As can be seen, both results are almost identical. Investigated further one can find that the average difference between both solutions is less than $0.67 \%$. However, regarding the computation time, we found that the FEM is faster then the hybrid method for about $77 \%$.

After we proved that the hybrid method could be used for magnetostatic computations with the same accuracy as the ordinary FEM, we proceed with the main investigation; that is, solution of the moving coil problem using the hybrid method. For that purpose, we moved a coil arbitrary inside the EFGM area (dark gray area) and repeat the computation without any remeshing of the model. The results show that with minimal efforts we were able to perform an accurate analysis of the problem. If we used the FEM, then we had to remesh the coil and its surrounded area for each analysis. It is obvious that this makes an ordinary FEM analysis difficult and unsuitable for a class of problems with moving objects.

\section{Inverse Shape Optimization Problem}

Inverse shape optimization problems are usually very CPU and computer resources consuming analyzes because for each intermediate solution a computation or evaluation of the quality of the obtained solution must be performed. In $2 \mathrm{D}$ analysis, the process of remeshing of the analysis domain is usually more time consuming than the actual computation process. Therefore, the main objective for the development of the hybrid method was to decrease the computational difficulties and to increase the computational speed of the inverse optimization processes.

A model of a C-type electromagnet shown in Fig. 9 was used for inverse shape optimization. Two models of the pole 


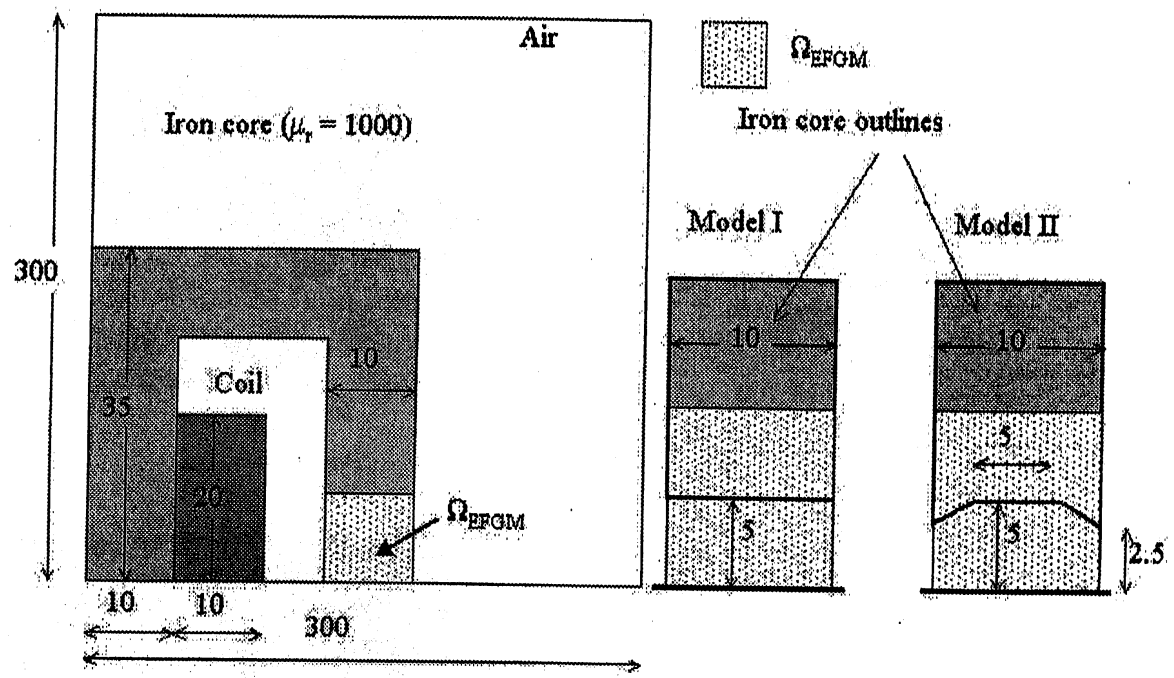

Fig. 9. Optimization model of a C-type electromagnet.
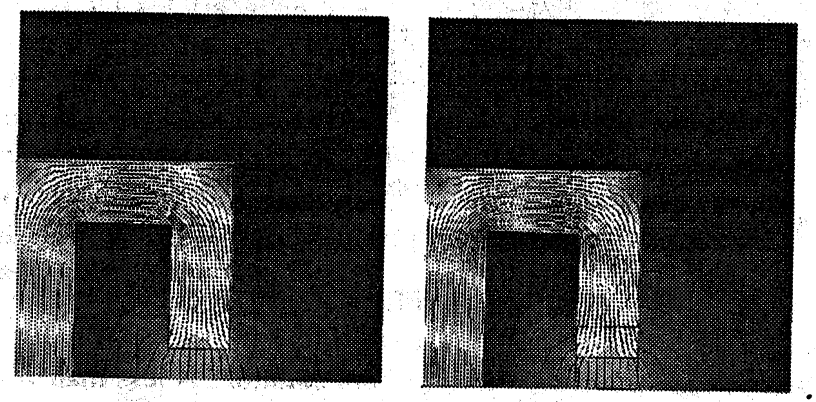

Fig. 10. Magnetic flux distribution and flux lines before optimization-Model I.

shape are also shown in Fig. 9: initial one and an intermediate one. Figs. 10 and 11 show the results comparison between the numerically obtained magnetic flux lines for the initial pole shape and for one intermediate pole shape of the model using the FEM and the proposed hybrid model, respectively. From both figures, it is apparent that compared magnetic flux distributions are almost identical for both methods. The maximum and the average differences between results obtained with both solution methods are given in Table III, while the computation time and the number of the ICCG iterations for the solution of the problem are given in Table IV. It is clear that the hybrid method for this problem is faster than the FEM, mainly because it needed less number of the ICCG iterations for its solution. Moreover, using the proposed hybrid method, inside the EFGM area that is around the pole face, a nodal distribution was generated only once (see Fig. 9). If we use the FEM, remeshing of the coil area and its surroundings must follow each change of the pole shape.

\section{CONCLUSIONS}

A new hybrid method for electromagnetic field computation is presented. This new method is based on the so-called meshless method, EFGM and the ordinary FEM. The entire anal-
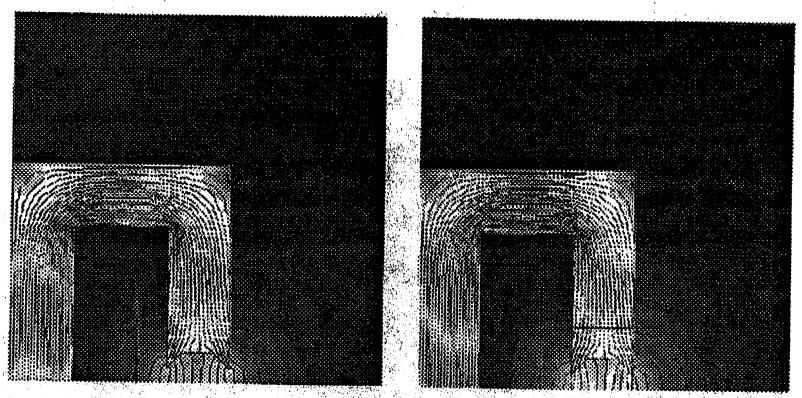

Fig. 11. Magnetic flux distribution and flux lines during optimization-Model II.

TABLE III

DIFFERENCES BETWEEN HYBRID METHOD AND FEM SOI. (TIONS[\%]

\begin{tabular}{lrr}
\hline & Model I & Model II \\
\hline Maximum difference & 2.73 & 9.12 \\
\hline Average difference & 0.23 & 0.59 \\
\hline
\end{tabular}

TABLE IV

COMPUTATION TIME-MODHI. I

COMPUTATION TiME-MODHi. II

\begin{tabular}{lrrr}
\hline & CPU time [sec] & Iterations \\
\hline Hybrid Method & 1.40 & 52 \\
\hline FEM & 2.74 & 381 \\
\hline & & \\
\hline & & \\
\hline Hybrid Method & CPU time [sec] & Iterations \\
\hline FEM & 1.41 & 52 \\
\hline
\end{tabular}

ysis domain is initially divided into three separate subdomains: EFGM subdomain, FEM subdomain, and an intermediate subdomain that ensures interconnectivity between the former two. Additionally, a new type of shape functions was also defined in order to ensure continuity between the solutions from both 
subdomains, the EFGM and the FEM subdomains. The proposed hybrid method was successfully applied for magnetostatic field computation where it shows the same order of accuracy as the ordinary FEM. Although computationally slower than the FEM, the proposed method could find applications for analysis of some special electromagnetic problems, such as problems that comprise moving objects, or problems where part of the domain changes its geometrical shape or disposition, as in cases of inverse shape optimizations.

\section{REFERENCES}

[1] V. Cingoski, N. Miyamoto, K. Kaneda, and H. Yamashita, "Element-free Galerkin method for electromagnetic field computations," IEEE Trans. Magnetics, vol. 34, no. 5, pp. 3236-3239, 1998.

[2] T. Nagashima, H. Okuda, and G. Yagawa, "Basic study on element-free Galerkin method: 2nd report, application to two-dimensional potential problem" (in Japanese), Transaction on Japanese Society of Mechanical Engineerings, vol. 62, no. 599, pp. 1746-1753, 1996.

[3] C. Herault and Y. Marechal, "Galerkin meshless methods: Implementation methodologies," in Proceedings of CEFC'98, Tucson, AZ, 1998, $\mathrm{p}$. 241.

[4] - "Boundary and interface conditions in meshless methods," IEEE Trans. Magnetics, vol. 35, no. 3, pp. 1450-1453, 1999. 